\title{
The Next Steps for Endocrine Pathology
}

\author{
Sylvia L. Asa ${ }^{1}\left(\mathbb{D} \cdot\right.$ Lori A. Erickson $^{2}\left(\mathbb{D} \cdot\right.$ Ozgur Mete $^{3}(\mathbb{D}$
}

Accepted: 19 February 2022 / Published online: 28 February 2022

(c) The Author(s), under exclusive licence to Springer Science+Business Media, LLC, part of Springer Nature 2022

Tremendous discoveries made over the last 30 years have advanced our understanding of many important aspects of the genetics, proteomics, prognosis, and therapy of endocrine neoplasia. These form the basis of the $2022 \mathrm{WHO}$ classification of endocrine and neuroendocrine tumors. However, there are still many unanswered questions and many unmet needs for patients. This commentary summarizes some of the questions and challenges for endocrine pathologists to address in the coming 5 years.

The pituitary is a model of neuroendocrine neoplasia that has been the subject of intense investigation. We have made important advances in understanding the cytogenesis of adenohypophysial cells and creating a detailed but clinically relevant classification of pituitary neuroendocrine tumors (PitNETs). Despite the progress made in the field, we are limited in our ability to predict which PitNET will behave aggressively and/or metastasize. It remains to be determined whether there is a high-grade primary pituitary neuroendocrine carcinoma (NEC) and if so, how to distinguish it from a PitNET with high-grade proliferative features. A PitNETspecific TNM staging system is needed to provide better prognostic information for locally invasive and metastatic lesions. As with other neoplasms, prevention of PitNETs remains an important goal. Only a small minority of these tumors is associated with germline genetic predisposition and the vast majority of sporadic tumors have no known genetic basis. The pituitary is responsible for integrating signals from our environment to facilitate adaptive mechanisms; as such, it is subject to epigenetic modifications that modulate the adaptive response. Numerous studies have

Sylvia L. Asa

Pathlady01@gmail.com

1 Department of Pathology, University Hospitals Cleveland Medical Center, Case Western Reserve University, Cleveland, OH, USA

2 Department of Laboratory Medicine and Pathology, Mayo Clinic, Rochester, MN, USA

3 Department of Pathology, University Health Network, University of Toronto, Toronto, ON, Canada shown that PitNETs are characterized by significant epigenetic alterations. Those who see patients with these tumors know well that the onset of disease can frequently be associated with a stressful life event and there have been efforts to uncover common mediators of hormonal and emotional responses to stress [1]. Interestingly, a recent study showed that alcohol consumption is associated with reduced risk of PitNET diagnosis [2], suggesting that reducing stress may prevent adenohypophysial neoplasia. The next 5 years will hopefully see more studies on the role of the environment in the development of PitNETs.

Neuroendocrine neoplasms of the gastroenteropancreatic system (GEP-NENs) are increasing in incidence. Mutations in genes that underlie neuroendocrine tumors (NETs) in multiple sites are being recognized as new familial syndromes emerge; however, the etiology of the more common sporadic tumors remains enigmatic. Several scenarios point to the role that neuroendocrine cells play in the balance between physiologic regulation and the environment; for example, gastric NETs can be a response to acidity that is altered by diet, alcohol, and drugs. While the etiologic mystery of multifocality of EC cell tumors of the small bowel requires further clarification, these tumors have proven to be almost devoid of mutations and instead harbor epigenetic changes, implicating environmental changes such as diet or the microbiome in their development. This concept can also be expanded to the respiratory system where the most aggressive NENs are known to be associated with the toxicity of smoking; there is work to be done on the factors associated with the development of low-grade NETs in the lungs. The next 5 years should see a focus on prevention as we further our understanding of the importance of environmental changes on neuroendocrine homeostasis.

The development of new biomarkers allows the pathologist to identify not only the neuroendocrine nature of a neoplasm, but also its site of origin and hormonal function. The concept of a tumor of unknown primary site is rapidly disappearing as we apply these new tools. The application of Ki67 as a determinant of proliferative grade is becoming more widely adopted and the use of algorithms to enhance 
the speed and accuracy of the tool is improving the ability to replicate outcomes, but this is still a limited grading system that uses a continuous variable with artificial cut-off points. As shown in the pituitary, pancreas, and rectum, more sophisticated studies are needed to move the classification to the next level based on cell type and hormone profiles.

The classification that divides NETs from NECs has been widely adopted based on the genetic alterations that are usually distinct in these two types of NENs. However, as in any classification, there are exceptions and borderline cases. Given the evidence for tumor progression by accumulation of additional genetic alterations, it seems likely that at least some large cell NECs may not represent true primary NECs but rather may be examples of progression of a NET [3]. There is clearly a need for more and better biomarkers to distinguish high-grade NETs from NECs and an expanded focus on observational correlates of the NET-to-NEC progression sequence. Similarly, the distinction of carcinomas with neuroendocrine trans-differentiation from amphicrine malignancies requires further clarification.

The expanding spectrum of familial endocrine neoplasia syndromes will allow us to better screen for early neoplasia and prevent disease. The perfect example of this is MEN2 where we have well-defined genotype-phenotype correlations that provide guidance for the timing of prophylactic thyroidectomy to ensure excellent patient outcomes. However, this example of inheritance of a mutated activated oncogene is unique; every other inherited tumor syndrome involves loss of a tumor suppressor gene and we have no ability to clearly predict the timing and location of the necessary "second hit" that will result in tumorigenesis. The recognition of precursor lesions in many of these familial disorders and the application of molecular immunohistochemistry, as detailed in many of the papers in this issue, provide pathologists with opportunities to predict the need for molecular testing early in the course of the disease. As in the case of paragangliomas, where catecholamine metabolites provide excellent biochemical clues, we must pursue the hormones and other circulating biomarkers that will allow us to determine when a patient requires intervention for epithelial NENs. Improvements in imaging techniques, such as $\mathrm{Ga}^{68}$-DOTATATE PET scans, allow accurate localization of small lesions to facilitate targeted cures, and the idea of using early peptide receptor radiotherapy (PRRT) for this goal, rather than relegating it as a salvage therapy for widely disseminated disease, deserves consideration. The application of FDG-PET is gradually seeing increasing recognition as a tool to identify aggressive NETs.

The most common endocrine pathology seen by the surgical pathologist involves the thyroid where we see a huge volume of benign disease, fortunately very rare highly aggressive malignancy, and a full spectrum of intermediate lesions that prove the stepwise progression of neoplasia associated with accumulation of additional genetic events. Molecular pathology has clarified many misconceptions in this field; however, there are still many barriers to a logical Linnaean classification of thyroid tumors. The last decade has provided a clear picture of the BRAF-like basis of papillary carcinoma and the RAS-like basis of follicular neoplasms. This is slowly opening the door to a clearer concept of two main families of thyroid follicular cell neoplasia and the opportunity to dispel the myth of nuclear features as a basis for subclassification; however, it is hard to unlearn many of the traditional teachings. The 5th edition of the WHO is paving the way for the acceptance of "papillary adenoma" and the unification of follicular carcinoma with follicular variant papillary carcinoma. The basis of separating oncocytic thyroid neoplasms from their nononcocytic counterparts is still an area that requires careful consideration given the heterogeneity in diagnostic criteria applied. While the concept of mitoses and necrosis as features of high-grade differentiated follicular cell neoplasms is now accepted, the classification of differentiated thyroid carcinoma with a mitotic count of 3 or 4 per $2 \mathrm{~mm}^{2}$ remains unanswered in the 2022 WHO classification. As we elucidate the biomarkers that predict local and distant spread, we can better stratify treatment approaches and be clear about the distinction between low- and high-risk malignancy, without resorting to "indeterminate" and "uncertain" diagnoses. The next 5 years should see progress in how thyroid neoplasia is classified based on tumor growth patterns, invasion, nuclear atypia, translational biomarkers, and other risk factors.

Advances in the field of parathyroid and adrenal cortical tumors have significantly impacted the way that endocrine pathologists diagnose these tumors and have prognostic value. The definition and value of angioinvasion as a diagnostic and prognostic feature is becoming more widely accepted. When dealing with borderline lesions, the application of multifactorial parametric scoring systems remains a primitive approach to adrenal cortical neoplasia that should be replaced by translational biomarkers that allow early detection of malignancy. These changes are required to shape the next level of diagnostic pathology practice, and we hope it will impact the 6th edition of the WHO classification.

These are just a few of the unanswered questions and challenges that arise as one studies the 5th edition WHO Classification of Endocrine and Neuroendocrine Tumors.

Author Contribution Conception and design: SLA, OM; manuscript preparation and editing: SLA, OM; approval of final manuscript: SLA, OM, LAE. 


\section{Declarations}

Ethics Approval Not applicable.

Consent for Publication All authors consent to publication.

Competing Interests Dr. Ozgur Mete is the Editor-in-Chief of Endocrine Pathology. Dr. Lori Erickson is the Senior Associate Editor of Endocrine Pathology. This article was handled by an independent editor and peer-reviewed as per the journal standards.

\section{References}

1. Kiehl TR, Fischer SE, Ezzat S, Asa SL (2008) Mice lacking the transcription factor Ikaros display behavioral alterations of an anti-depressive phenotype. Exp Neurol 211:107-114.
2. Cote DJ, Smith TR, Kaiser UB, Laws ER, Jr., Stampfer MJ (2022) Alcohol intake and risk of pituitary adenoma. Cancer Causes Control 33: 353-361. https://doi.org/10.1007/s10552-021-01523-0

3. Pelosi G, Bianchi F, Dama E, Metovic J, Barella M, Sonzogni A, Albini A, Papotti M, Gong Y, Vijayvergia N (2021) A Subset of Large Cell Neuroendocrine Carcinomas in the Gastroenteropancreatic Tract May Evolve from Pre-existing Well-Differentiated Neuroendocrine Tumors. Endocr Pathol 32: 396-407. https://doi. org/10.1007/s12022-020-09659-6

Publisher's Note Springer Nature remains neutral with regard to jurisdictional claims in published maps and institutional affiliations. 